Classification

Physics Abstracts

$61.16 \mathrm{D}-81.30$

\title{
Phase Identification in Carbon and BN Systems by EELS
}

\author{
Herbert K. Schmid \\ Div. Materials Science and Technology, CSIR, Pretoria/RSA, South Africa
}

(Received July 27; accepted November 23, 1994)

\begin{abstract}
Electron energy-loss spectroscopy measurements have been made on carbon and BN polymorphs, as well as on a number of borides, carbides and nitrides present in sintered $\mathrm{cBN}$ based compacts. The similarity in crystal symmetry between carbon and the isotructural $\mathrm{BN}$ system results in spectral similarities in both the low-loss features and electron energy-loss near-edge structure associated with the particular core-loss ionization edges. ELNES features hold information on the nearest neighbour environment of the excited atom in a given phase. It is shown that the analysis of these features provides characteristic coordination fingerprints for the identification of carbon and $\mathrm{BN}$ allotropes. Similarly, the comparison of ELNES features associated with B-K, C-K and N-K ionization edges provides fingerprints for the identification of $\mathrm{B}$ in $\mathrm{TiB}_{2}$ and $\mathrm{B}_{4} \mathrm{C}, \mathrm{C}$ in $\mathrm{TiC}$ and $\mathrm{B}_{4} \mathrm{C}$, and $\mathrm{N}$ in AIN, TiN and BN, respectively. An attempt is made to quantify the fraction of $\mathrm{sp}^{3}$ bonding in diamond-like carbon and $\mathrm{cBN}$ based cutting tool material from the relative intensity of the $\pi$ resonance in C-K and B-K ELNES, respectively.
\end{abstract}

\section{Introduction}

Superhard materials are of increasing technological interest, both in form of bulk compacts as well as thin films and coatings. Diamond films (monocrystalline/polycrystalline diamond (PCD), amorphous diamond-like carbon (DLC)) are of particular interest for tribological and electronic applications because of their extreme hardness combined with electronic insulation and thermal conductivity characteristics. The carbon and boron nitride (BN) systems are isoelectronic/isostructural and therefore similar allotropic forms exist in both systems, i.e. the high pressure phases, cubic diamond (cD) and cubic BN (cBN), the hardest known materials both crystallizing in a sphalerite type (zinc blende) lattice; the metastable high pressure phases, hexagonal diamond (hD, lonsdaleite) and wurtzitic $\mathrm{BN}(\mathrm{wBN})$; and the low pressure phases, graphite (gC) and graphitic $\mathrm{BN}$ (hexagonal hBN). Optimization of materials properties requires a precise characterization in terms of microstructure/microchemistry, crystallography and identification of the diamond structure, characterized by $\mathrm{sp}^{3}$ hybridization of the $\mathrm{C}-\mathrm{C}$ and $\mathrm{B}-\mathrm{N}$ bonds in tetrahedral coordination, in particular. Because of its strong covalent nature, sintering of $\mathrm{cBN}$ based dense compacts requires the addition of carbide and/or nitride binder phases (e.g. TiC, $\mathrm{HfC}, \mathrm{AlN}$ ) which during thermal processing may form secondary inter-reaction phases such as $\mathrm{TiB} / \mathrm{TiB}_{2}, \mathrm{TiN}, \mathrm{AlB}_{2}, \mathrm{~B}_{4} \mathrm{C}$ etc. by 
reacting with the matrix constituents. Optimization of such complex heretostructures requires their precise characterization in terms of phase identification, phase distribution and morphology in order to understand their properties as a result of the microstructural evolution process.

Electron energy-loss spectroscopy (EELS) constitutes an indispensable tool for quantitative light element chemical analysis with high spatial resolution. However, valuable additional chemical and structural information may be obtained from the analysis of both the low-loss features (plasmon excitation and interband transitions) as well as the electron energy-loss near edge structure (ELNES) associated with each high-energy core-loss ionization edge. Since the plasmon energy is a function of the carrier charge density [1] it may hold information on both the mass density (and thus the structure and nature of chemical bonds) and valency state of the target material [2]. ELNES features essentially involve transitions of core electrons to unoccupied states just above the Fermi level and thus may hold information on the density of final states, the electronic structure, site symmetry and nearest neighbour coordination of the excited atom in a given phase. In many cases it is found that the analysis of such structure specific so-called coordination fingerprints $[3,4]$ may allow the identification of unknown phases in complex systems containing borides, nitrides, carbides or oxides in particular.

In the present work low-loss and core-loss ELNES features observed in the isostructural carbon and BN systems, as well as fine structures of $\mathrm{K}$-edges of some borides, carbides and nitrides relevant for the characterization of $\mathrm{cBN}$ based heterostructures are analyzed. It is aimed to build up a reference library of ELNES fingerprints for the rapid phase identification in these systems. This is of particular interest for the characterization of diamond, DLC and BN thin film coatings as well as nanocrystalline bulk heterostructures in terms of crystallography, phase identification and $\mathrm{sp}^{2} / \mathrm{sp}^{3}$ bonding character.

\section{Results and Discussion}

2.1 EXPERIMENTAL DETAILS. - Electron transparent self-supporting foils of BN compacts were prepared using standard preparation methods for ceramic materials, i.e. mechanical dimple grinding/polishing followed by Ar ion-beam etching. Specimens of diamond and BN coatings have been prepared either in plan-view by back-thinning, or in cross-section. Hexagonal diamond and wBN powder particles, were dusted onto holey carbon films, only very small particles protruding over the edge of the supporting carbon film were selected for EELS analysis. The relative specimen thickness $t / \lambda$ (with $\lambda$ the mean free path for inelastic scattering), which is related to the extent of plural scattering, was estimated from the intensity ratio $I_{\mathrm{p}} / I_{\mathrm{o}}$ where $I_{\mathrm{p}}$ is the intensity of the inelastically scattered low-loss electrons essentially contained in the plasmon peaks ( $3 \leq \Delta E<100 \mathrm{eV}$ ), and $I_{\mathrm{o}}$ is the intensity of the zero-loss peak. The effects of plural scattering in core-loss spectra have been removed by applying a Fourier-Ratio deconvolution procedure [1].

EEL spectra were acquired with a GATAN 607 spectrometer attached to a Philips EM 420 TEM/STEM fitted with an $\mathrm{LaB}_{6}$ filament. The spectra were recorded in TEM/STEM diffraction mode operated at $120 \mathrm{kV}$ primary voltage. Using a $2 \mathrm{~mm}$ spectrometer entrance aperture yielded an energy resolution $<2 \mathrm{eV}$. The effective spectrometer acceptance angle was varied in the range $3 \leq 2 \beta \leq 8 \mathrm{mrad}$ by selecting appropriate camera lengths in order to optimize the signal/background ratio [5].

2.2 The CARbon SYSTEM. - EEL spectra from a variety of carbon modifications were acquired under comparable conditions [6]. The spectral features of interest in the low-loss region $0 \leq \Delta E \leq 90 \mathrm{eV}$, and the core-loss region $280 \leq \Delta E \leq 330 \mathrm{eV}$ around the $\mathrm{C}-\mathrm{K}$ ionization edge from cubic diamond (De Beers, Johannesburg), hexagonal diamond (Du Pont, Gibbstown, 
NJ), amorphous diamond-like carbon (AEC, Pretoria) and partially graphitized carbon (Polaron, Watford) are shown in Figure 1. In the (poly) crystalline carbon modifications no specific crystal orientations were selected and strong Bragg reflections were avoided in single crystalline cD particles by tilting the specimen away from Bragg orientations for the acquisition of the EEL spectra.
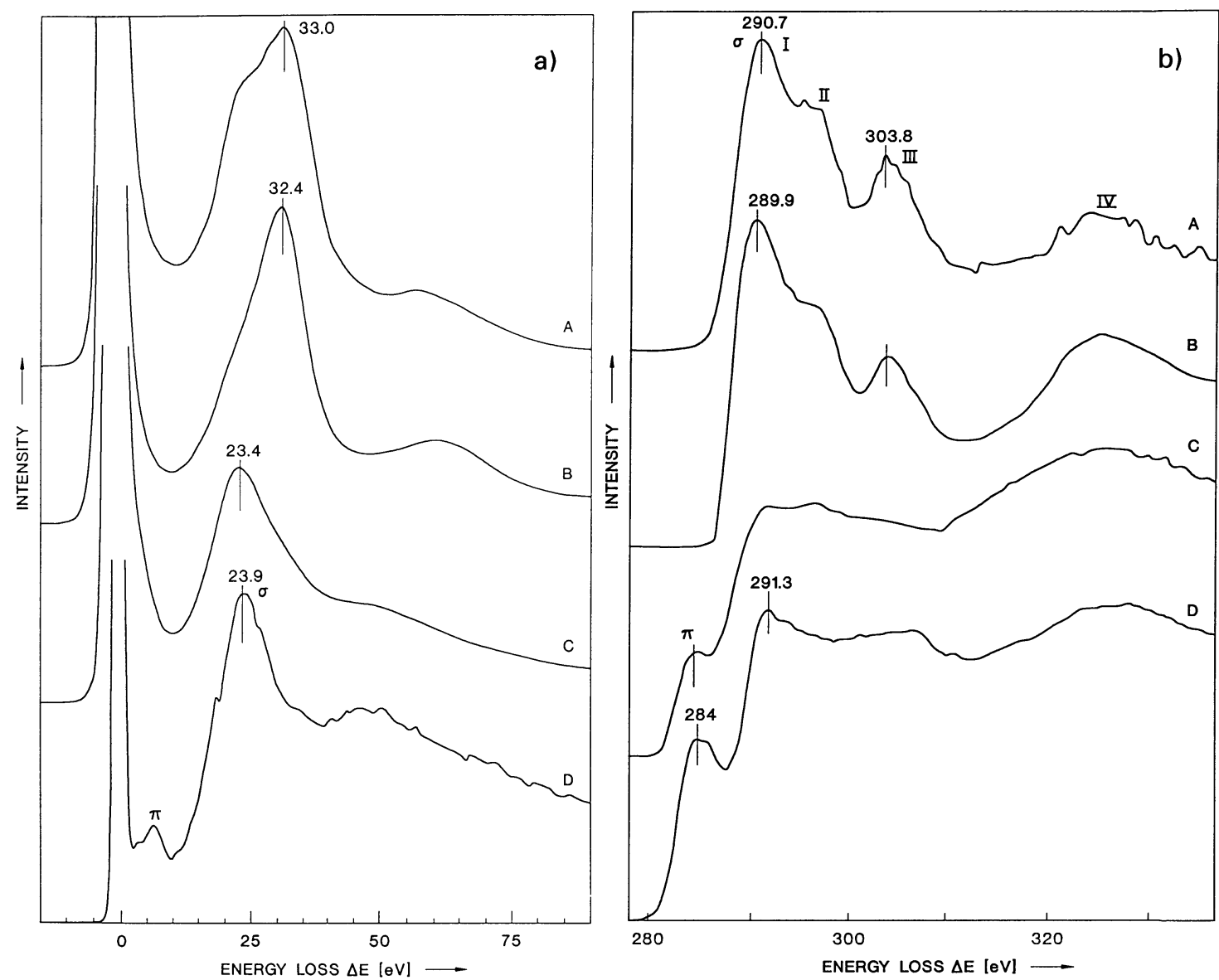

Fig. 1. - EEL spectra from cubic diamond (A), hexagonal diamond (B), diamond-like carbon (C), and partially graphitized carbon (D); a) low-loss region dominated by zero-loss peak and plasmon peak; b) coreloss ELNES associated with C-K ionization edge (stripped edges).

The low-loss region:

The plasmon peaks associated with the collective excitation of the bulk valence electrons are shown in Figure 1a. The main plasmon peak of $\mathrm{cD}(\mathrm{A})$ is centred at $33 \mathrm{eV}$ and shows a prominent shoulder at the low-energy slope at $\approx 23.5 \mathrm{eV}$. This hump, not observed in the spectrum (B) from $\mathrm{hD}$ seems to be a characteristic of the cubic diamond structure [7]. Since the relative specimen thickness of the $\mathrm{cD}$ sample $(t / \lambda=0.37)$ was larger than of the $\mathrm{hD}$ crystallite $(t / \lambda=0.32)$ surface effects can be ruled out and the additional intensity contribution around $\Delta E \approx 23 \mathrm{eV}$ may be attributed to interband transitions in the cubic polymorph [8]. The plasmon peak from $\mathrm{hD}$ (spectrum $\mathrm{B}$ ) is slightly shifted to lower energy, peaking at $32.4 \mathrm{eV}$; the marginal change in 
slope observed at the low-energy side around $\approx 20 \mathrm{eV}$ could be a contribution from some $\mathrm{cD}$ present in this material since lonsdaleite is not synthesized as a pure phase by dynamic shock wave-conversion of hexagonal graphite [9].

The plasmon peaks from DLC (spectrum C) and graphitized carbon (gC, spectrum D) are centred around $\approx 24 \mathrm{eV}$. In addition to $\sigma$ plasmons the spectrum (D) recorded at an energy resolution of $1 \mathrm{eV}(2 \beta=1.5 \mathrm{mrad})$ shows a peak at $6.4 \mathrm{eV}$ attributed to $\pi \rightarrow \pi^{*}$ transitions; minor peaks observed at $\approx 3.5$ and $18.4 \mathrm{eV}$ can be assigned to interband transitions [10]. According to a free electron gas model of plasmon oscillations for the valence electrons, the energy $E_{\mathrm{p}}$ of the bulk plasmon mode is given by

$$
E_{\mathrm{p}}=\hbar\left[n_{\mathrm{c}} e^{2} / m^{*} \varepsilon_{\mathrm{o}}\right]^{1 / 2}
$$

where $\varepsilon_{\mathrm{o}}$ is the dielectric function, $m^{*}$ and $e$ the effective electron mass and charge, and $n_{\mathrm{c}}$ the carrier charge density [1]. Since $n_{\mathrm{c}}$ is a function of both the valency state and the mass density $\rho, E_{\mathrm{p}}$ is expected to show a strong dependency on $\rho$ as seen in Figure 1a. The slightly reduced plasmon energy in $\mathrm{hD}$, compared with $E_{\mathrm{p}}$ in $\mathrm{cD}$, can be interpreted as an indication of a lower mass density due to the presence of lattice defects in this metastable material. Similarly, it can be argued that the DLC films analyzed in these studies might have low apparent mass density possibly due to both its amorphous nature and a high concentration of hydrogen in this material, resulting in reductions of the mean number of valence electrons and the average atomic weight. While the $\sigma$ plasmon in DLC resembles that of $\mathrm{gC}$, no indication on the presence of $\pi$ plasmons was observed in DLC, similar to observations in amorphous carbon [7, 10].

\section{The high-energy core-loss region:}

The C-K ionization edges from the same four modifications of carbon are shown in Figure $1 \mathrm{~b}$. For clarity the background was stripped from the original spectra (inverse power law background modelling) and plural scattering contributions were removed by a Fourier-Ratio deconvolution procedure. The most prominent features seen in diamond (spectra A and B) are a sharp rise in intensity at the edge onset at $287 \mathrm{eV}$, followed by a series of peaks labelled I, II, III and IV respectively, assigned essentially to $1 \mathrm{~s} \rightarrow \sigma^{*}$ transitions in $\mathrm{sp}^{3}$ hybridized carbon [11], whereas the $\pi$-peak characteristic of $\mathrm{sp}^{2}$ bonding is completely absent. The ELNES features observed in spectra A and $B$ appear to be very similar to each other, the only noticeable difference is an increased peak intensity ratio $I_{\mathrm{IV}} / I_{\mathrm{III}}$ of spectrum B in comparison with A. Since plural scattering contributions have been removed, the remaining effect is attributed to originate from a highly disordered defect structure of $\mathrm{hD}$. This view seems to be supported by the observation of a prominent broad peak centred at $\approx 325 \mathrm{eV}$ in spectra from amorphous diamond $(\mathrm{aD})$ [7], corresponding with peak IV whereas peak III seems to be absent in $\mathrm{aD}$ and of reduced intensity in disordered $\mathrm{hD}$. This is also in accordance with our observations in DLC (spectrum C) which show a broad peak IV of relatively high intensity; peak II is marginally visible whereas peak III is flattened out. A number of EELS studies on various forms of carbon are reported in the literature $[12,13]$ which infortunately excluded the energy range $\Delta E>310 \mathrm{eV}$, thus precluding a direct comparison with above observations.

A comparison of the spectra from DLC (C) and $\mathrm{gC}(\mathrm{D})$ shows a residual feature at $284 \mathrm{eV}$ in DLC corresponding to $1 \mathrm{~s} \rightarrow \pi^{*}$ transitions, confirming the presence of some $\mathrm{sp}^{2}$ bonded carbon in DLC. The spectrum (D) from partially graphitized carbon (gC) was collected from a specimen area containing paracrystalline graphite particles at random orientation. Electron diffraction patterns indicated a considerable fraction of amorphous carbon present in this sample material. The $\pi$-peak at $\Delta E \approx 284 \mathrm{eV}$, characteristic of $\mathrm{sp}^{2}$ bonding, is clearly resolved. 
2.3 THE BORON NiTRIDE SYSTEM. - Carbon and boron nitride (BN) systems are isoelectronic and therefore corresponding polymorphs described for carbon also exist in the BN system, i.e. $\mathrm{cBN}$, wBN and graphitic $\mathrm{hBN}$. Though the properties of corresponding $\mathrm{C}$ and $\mathrm{BN}$ allotropes are essentially similar, there are also differences due to the $\approx 20 \%$ ionic character of the B-N bond. Consequently $\mathrm{BN}$ attracts much interest because of its unique combination of properties such as extreme hardness (cBN/wBN), high anisotropy (hBN), low specific mass and high chemical stability, of which high temperature oxidation resistance and chemical inertness to ferrous metals are of particular interest. The characteristic features seen in EEL spectra from cBN (De Beers, Johannesburg), wBN (Inst. Superhard Materials, Kiev/Ukraine), cBN based cutting tool materials (Sumitomo, Japan) and hBN (BN/TiB 2 composite, CSIR, Pretoria) are shown for comparison in Figure 2.
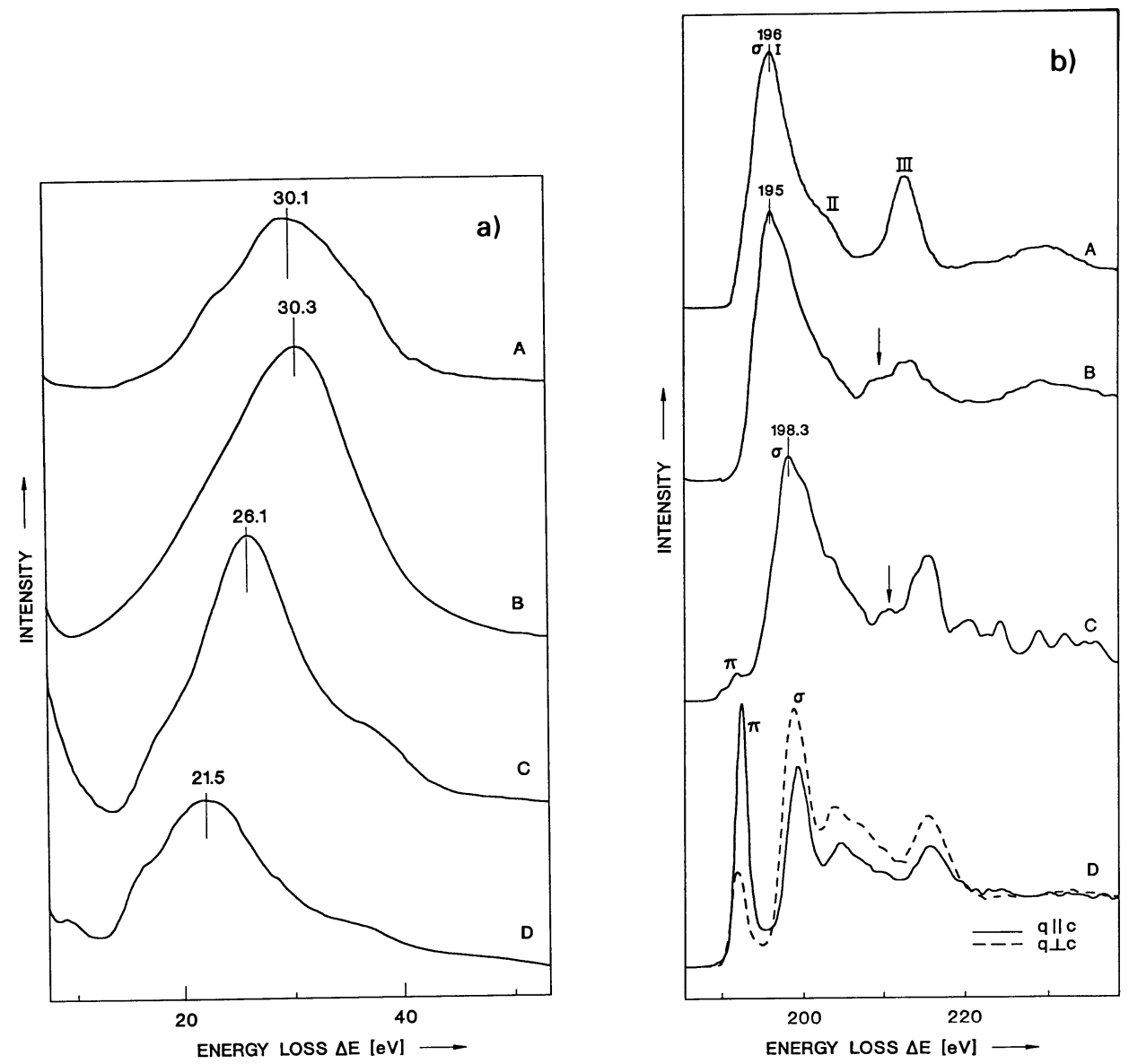

Fig. 2. - EEL spectra from cubic BN (A), wurtzitic BN (B), cBN based cutting tool material (C), and hexagonal BN (D); a) low-loss region; b) core-loss ELNES associated with B-K ionization edge; momentum transfer vector $(q \| c)$ parallel, and $(q \perp c)$ perpendicular to $c$-axis, respectively.

The low-loss region:

The low-loss features seen in EEL spectra from above BN allotropes in the energy range 
$<50 \mathrm{eV}$ are dominated by the bulk plasmon peaks as shown in Figure 2a. The spectrum (A) from $\mathrm{cBN}$ shows a characteristic hump at the low-energy slope of the main peak at $\approx 22 \mathrm{eV}$, very similar to the observation in $\mathrm{cD}$; again this feature is not observed in spectrum (B) from wBN. The major plasmon peaks in both $\mathrm{cBN}$ and $\mathrm{wBN}$ are shifted to lower energies $\approx 30 \mathrm{eV}$ in comparison with diamond $(\approx 33 \mathrm{eV})$. Similarly, the $\sigma$ plasmon peak centred at $\approx 21.5 \mathrm{eV}$ in hBN is shifted to lower energy in comparison with graphitic carbon $(\approx 24 \mathrm{eV})$, again a $\pi$ peak at $\approx 7 \mathrm{eV}$ is clearly visible. The plasmon peak from $\mathrm{BN}$ in the binder phase of a $\mathrm{cBN}$ based cutting tool material (spectrum C) was observed to be centred at a position between the peak positions in $\mathrm{cBN}$ and $\mathrm{hBN}$, suggesting a heterophase material of lower mass density (in comparison with $\mathrm{cBN}$ ) composed of $\mathrm{hBN}$ and/or $\mathrm{cBN} / \mathrm{wBN}$.

\section{High-energy core-losses:}

The B-K ionization edges of the BN allotropes are shown in Figure $2 \mathrm{~b}$. The similarity between the isostructural carbon and $\mathrm{BN}$ system results in spectral similarities, not only in the low-loss features but also in ELNES associated with core-losses. The spectra (A) and (B) from cBN and wBN are dominated by a strong $\sigma$ resonance centred at $\approx 196 \mathrm{eV}$ and $195 \mathrm{eV}$ followed by two major peaks labelled II and III, respectively. Comparison with Figure 1b shows that these two spectra are not only similar to each other but also to those spectra from the corresponding carbon allotropes $\mathrm{cD}$ and $\mathrm{hD}$. However, spectrum (B) from wBN shows a characteristic shoulder at the low-energy slope of peak III (indicated by an arrow). This feature, not observed in corresponding spectra from hD, may provide a clear ELNES fingerprint for an unambiguous identification of wBN [14].

Spectrum (D) from hBN is dominated by a characteristic $\pi$ peak centered at $\approx 191 \mathrm{eV}$ followed by $\sigma$ resonances I, II and III; it should be noted that peaks I and II are clearly separated in this structure. EEL spectra from graphitic $\mathrm{hBN}$ were obtained at two different orientations such that the crystal $c$-axis was either parallel or perpendicular to the incident electron beam and thus to the momentum transfer vector $q$. At $q \| c$ the momentum transfer is essentially in the direction of the $\pi$ bonds between adjacent (0002) basal planes, thus a strong $\pi$-peak is expected in this orientation. Accordingly at $q \perp c$ the major contribution to the ELNES should come from the $\sigma$ bonds between the nearest neighbours within the same basal plane, thus a stronger $\sigma$-resonance and a relatively weaker $\pi$-peak can be expected in this orientation, similar to observations in single crystalline graphite [15]. The spectrum (C) from the binder phase in $\mathrm{cBN}$ based material appears to be essentially a superposition of spectra $(A)$ and $\left(D_{q \perp c}\right)$. The shoulder observed at the low-energy slope of peak III (indicated by an arrow) could be interpreted as an indication for the presence of some wBN in the binder phase. Since BN is a minor inter-reaction phase in the binder of cutting tool materials (the majority being $\mathrm{TiC}, \mathrm{TiN}$ and $\mathrm{AlN}$ ) the $\mathrm{B}-\mathrm{K}$ signal-to-background ratio from the binder usually is rather low and noisy, and thus should not be overinterpreted. However, a $\pi$-peak indicative of the presence of some $\mathrm{sp}^{2}$ bonding in this material is clearly visible.

ELNES features associated with the N-K edges in hBN and wBN are shown in Figure 3 for comparison with spectra from other nitrides. The spectrum from hBN shows a $\pi$ peak at $\approx 400 \mathrm{eV}$ followed by two major $\sigma$ resonances, whereas in wBN (and similarly in $\mathrm{cBN}$ ) no $\pi$ peak and a single major $\sigma$ resonance is observed. A very weak shoulder seen at the high energy slope of this peak at $\approx 415 \mathrm{eV}$ might be a characteristic ELNES fingerprint of the wBN structure [14].

2.4 ELNES IN NITRIDES, CARBIDES AND BORIDES. - ELNES features associated with B-K, $\mathrm{C}-\mathrm{K}$ and N-K ionization edges in borides, carbides and nitrides relevant to the characterization of binder phases in $\mathrm{CBN}$ based superhard ceramic composites have been investigated with the aim of reliable phase identification based on characteristic fingerprints in these complex heterostructures [16]. The N-K edges from AlN, TiN, hBN and wBN are shown in Figure 3. In AlN the $\pi$ and $\sigma$ 


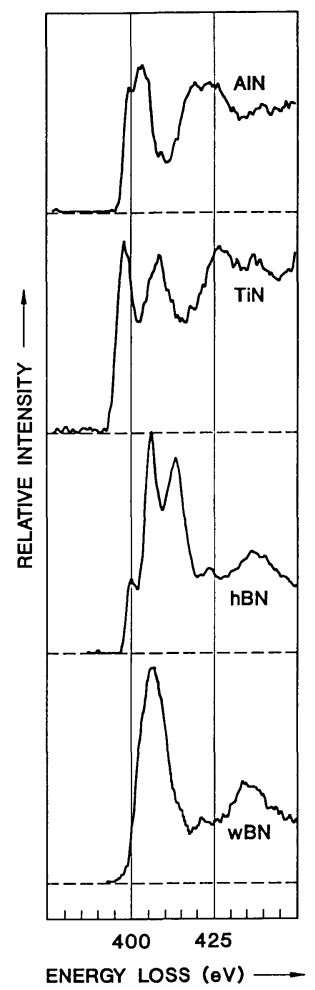

Fig. 3. - ELNES associated with N-K edge in AIN, TiN, hBN and wBN.

resonance are very close to each other at $\approx 398 \mathrm{eV}$ and $\approx 402 \mathrm{eV}$, whereas in TiN these peaks are well separated $(\approx 397 \mathrm{eV}$ and $\approx 407 \mathrm{eV}$, respectively). There is a noticeable similarity between the N-K ELNES of TiN and the C-K ELNES of TiC (Fig. 5) associated with the complex bonding structure in transition metal nitrides and carbides [17]. The N-K ELNES of hBN and wBN shown for comparison are distinctly different from those of the other nitrides; energy positions (peak maxima) of major peaks in borides, carbodes and nitrides relevant in this context are listed in Table I.

The B-K edges of $\mathrm{TiB}_{2}, \mathrm{~B}_{4} \mathrm{C}$ and Na-borate glass are shown in Figure 4. In $\mathrm{TiB}_{2}$ the $\pi$ and a broad $\sigma$ resonance are not well separated whereas in $\mathrm{B}_{4} \mathrm{C}$ the $\pi$ peak at $\approx 190 \mathrm{eV}$ is clearly resolved. The B-K ELNES in amorphous Na-borate is shown for comparison and differs considerably from those of $\mathrm{TiB}_{2}, \mathrm{~B}_{4} \mathrm{C}$ and $\mathrm{cBN} / \mathrm{wBN}$ polymorphs. The energy-loss and shape of the $\pi$ and $\sigma$ peaks at $\approx 191 \mathrm{eV}$ and $200 \mathrm{eV}$, respectively, show similarities with the ELNES seen in hBN (Fig. 2b), however peaks II and III are not observed in the amorphous structure due to the absence of long range order. It should be pointed out that the B-K ELNES in Na-borate glass differs considerably from that reported for amorphous BN [14]; it seems that the material termed amorphous BN appears to be rather composed of microcrystalline hBN.

Three C-K edges from $\mathrm{B}_{4} \mathrm{C}$, TiC and amorphous carbon $(\mathrm{aC})$ are shown in Figure 5 . The ELNES of TiC is dominated by well separated strong $\pi$ and $\sigma$ peaks of approximately equal intensity centred at $\approx 286 \mathrm{eV}$ and $294 \mathrm{eV}$ respectively. The presence of a $\pi$ resonance in C-K ELNES is characteristic of unsaturated $\mathrm{sp}^{2}$ (and in the case of aC also sp) carbon bonds. The overall shapes of $\sigma$ peaks in $\mathrm{B}_{4} \mathrm{C}$ and aC appear to be very similar, however in the former the $\pi$ peak is not 
Table I. - Energy losses of major ELNES features (peak centre position in eV) associated with B-K, $C-K, N-K$ and Ti-L ionization edges (averaged values obtained by multiple spectra analysis).

\begin{tabular}{|c|c|c|c|c|c|c|c|c|}
\hline $\begin{array}{l}\text { ELNES } \\
\text { Phase }\end{array}$ & $\pi$ & $\mathrm{K}_{\sigma}$ & $\pi$ & $\mathbf{K}_{\sigma}$ & & ${ }_{\sigma}$ & $\mathbf{L}_{3}$ & $-\mathbf{L}_{\mathbf{L}_{2}}$ \\
\hline $\begin{array}{l}\mathrm{cD} \\
\mathrm{hD} \\
\mathrm{aC} \\
\mathrm{cBN} \\
\mathrm{wBN} \\
\mathrm{hBN} \\
\mathrm{TiC} \\
\mathrm{TiN} \\
\mathrm{TiB}_{2} \\
\mathrm{~B}_{4} \mathrm{C} \\
\mathrm{AlN}\end{array}$ & $\begin{array}{l}- \\
190,7 \\
189,0 \\
189,8\end{array}$ & $\begin{array}{l}196 \\
195 \\
197,8 \\
\\
192,8 \\
198\end{array}$ & $\begin{array}{l}- \\
284 \\
285,8 \\
284,3\end{array}$ & $\begin{array}{l}290,7 \\
289,9 \\
295 \\
294 \\
291,2\end{array}$ & $\begin{array}{l}- \\
399,3 \\
397,4 \\
397,8\end{array}$ & $\begin{array}{r}403,9 \\
406,2 \\
406,3 \\
407,0 \\
401,7\end{array}$ & $\begin{array}{l}455,0 \\
455,7 \\
452,7\end{array}$ & $\begin{array}{l}459,6 \\
461,3 \\
458,2\end{array}$ \\
\hline
\end{tabular}

$\mathrm{cD}$ : cubic diamond; $\mathrm{hD}$ : hexagonal diamond (lonsdaleite); aC: amorphous carbon; cBN: cubic BN; wBN: wurtzitic BN; hBN: hexagonal BN

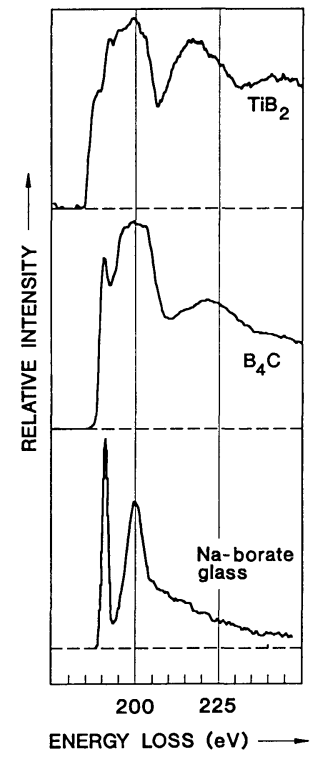

Fig. 4. - ELNES associated with $\mathrm{B}-\mathrm{K}$ edge in $\mathrm{TiB}_{2}, \mathrm{~B}_{4} \mathrm{C}$ and Na-borate glass.

well pronounced. In principle there would be a possibility to identify $\mathrm{TiC}$ by means of analysing the ELNES features associated with Ti-L ionization edges. However, this is severely hampered by phase overlap in nanocrystalline heterophase structures containing a variety of Ti compounds such as $\mathrm{TiC}, \mathrm{TiB}, \mathrm{TiB}_{2}$, TiN as well as mixed Ti-boride/nitride/carbides all giving rise to similar Ti-L ELNES [16].

2.5 QUANTIFICATION OF $\mathrm{sp}^{2} / \mathrm{sp}^{3}$ BONDING CHARACTER. - The crystalline modifications and compounds of carbon, analogous to the isoelectronic/isostructural $\mathrm{BN}$, can be classified into three 


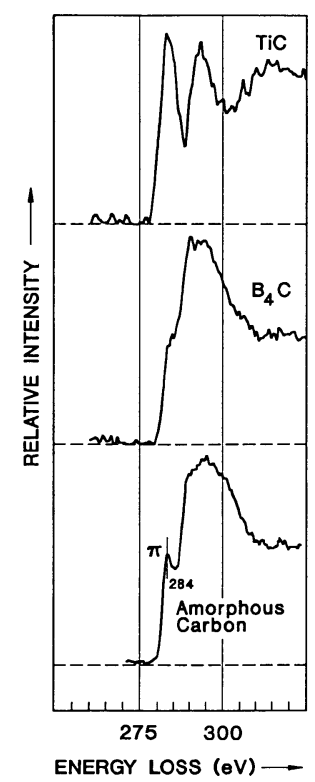

Fig. 5. - ELNES associated with $\mathrm{C}-\mathrm{K}$ edge in $\mathrm{TiC}, \mathrm{B}_{4} \mathrm{C}$ and amorphous carbon.

main structural forms with respect to the spatial distribution of strong directional covalent bonds. These are the linear form of carbides/borides, the lamellar form of graphite/graphitic hBN, and the tetrahedral form of diamond/cBN. Each form is characterized by the dominance of $\mathrm{sp}, \mathrm{sp}^{2}$ and $\mathrm{sp}^{3}$ bonding, respectively. It is essential to have a clear picture of the arrangement and bonding of the atoms in these structures since they determine the properties of the materials. In this context it is of particular interest to establish the crystallography and the extent of $\mathrm{sp}^{3}$ hybridization, which is characteristic of the diamond structure, in the various phases. An attempt was made to determine the ratio of $\mathrm{sp}^{2} / \mathrm{sp}^{3}$ bonding of carbon in DLC and of $\mathrm{BN}$ in the binder of a cBN based cutting tool material. Both the low-loss and core-loss features are related to the bonding character and may, in principle, be used for analysis [13].

According to equation (1) the square of the plasmon energy $\left(E_{\mathrm{p}}^{2}\right)$ is proportional to the charge density $n_{\mathrm{c}}$ and thus should be proportional to the effective mass density

$$
\rho=\frac{M n_{\mathrm{c}}}{n_{\mathrm{v}} N}
$$

where $M$ is the atomic weight, $n_{\mathrm{v}}$ the mean number of valence electrons per atom, and $N$ is Avogadro's number. The experimental values $\left(E_{\mathrm{p}}^{2}\right)$ as measured in EEL spectra from the BN and carbon allotropes versus their respective mass density $\rho$ are plotted in Figure 6; some data points from the literature $[7,14]$ are included for comparison. However, the data reported for $\mathrm{cBN}$ and wBN appear controversial. It would be difficult to explain a substantially higher plasmon energy in wBN, in comparison with $\mathrm{cBN}$, since the number of valence electrons per atom should be the same in both structures and furthermore the density of wBN is lower than that of $\mathrm{cBN}$ (3.45 versus $3.49 \mathrm{~g} \mathrm{~cm}^{-3},[18]$ ), thus a slightly lower carrier charge density $n_{\mathrm{c}}$ can be expected in wBN. The marginal discrepancy in $E_{\mathrm{p}}$ measured for $\mathrm{cBN}$ and $\mathrm{wBN}$ in the present work is well within the accuracy claimed $( \pm 0.5 \mathrm{eV})$. The experimental value $E_{\mathrm{p}} \approx 26.1 \mathrm{eV}$ measured in a cBN based cutting tool material suggest an apparent mass density $\rho \approx 2.86 \mathrm{~g} \mathrm{~cm}^{-3}$. Applying the law 
of mixtures, which states that $\rho$ is the summation of its constituent components weighted by their mole fractions, suggests a BN material composed approximately of $53 \% \mathrm{cBN} / \mathrm{wBN}$ and $47 \% \mathrm{hBN}$. This would correspond to an $\mathrm{sp}^{3}$ bond content of $\approx 53 \%$ by scaling the experimental plasmon energy to those of $\mathrm{hBN}\left(100 \% \mathrm{sp}^{2}\right)$ and $\mathrm{cBN}\left(100 \% \mathrm{sp}^{3}\right)$.

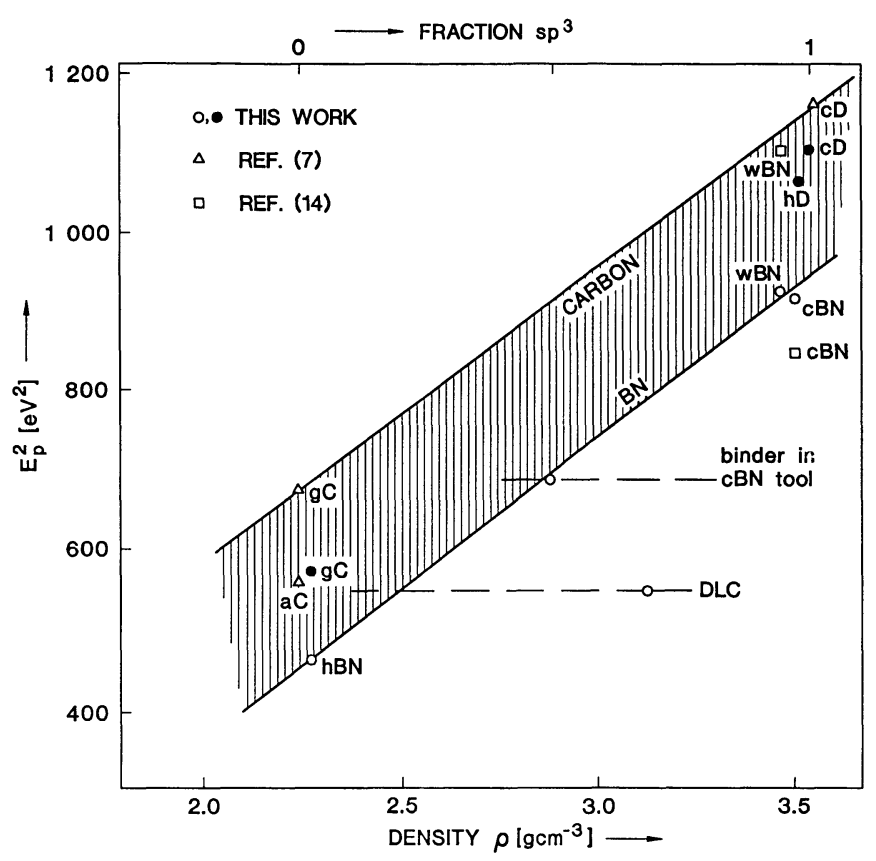

Fig. 6. - Summary of results from EELS analysis of low-loss features in carbon and BN systems.

The experiments confirmed that the plasmon energy losses in the carbon system tend to be systematically higher than in the $\mathrm{BN}$ system for both $\mathrm{sp}^{2}$ and $\mathrm{sp}^{3}$ bonded structures as shown in Figure 6. Assuming four valence electrons for each atom, equation (1) yields $E_{\mathrm{p}} \approx 31 \mathrm{eV}$ for diamond $\left(\rho=3.514 \mathrm{~g} \mathrm{~cm}^{-3}\right)$, and $\approx 20 \mathrm{eV}$ for graphite $\left(\rho=2.267 \mathrm{~g} \mathrm{~cm}^{-3}\right)$. It had been suggested that the higher experimental $E_{\mathrm{p}}$ values observed in the carbon system could be attributed to single-electron excitations resulting in an upward shift in the resonance energy by valenceconduction band transitions [7]; this should have a pronounced effect particularly in the diamond structure characterized by a wide band gap. Furthermore, the lower atomic weight of carbon $\left(C^{12.011}\right)$ compared with an average atomic weight of 12.41 in $\mathrm{BN}$, combined with slightly higher mass density due to shorter bond lengths in diamond $(0.154 \mathrm{~nm}$ in $\mathrm{cD}$, versus $0.157 \mathrm{~nm}$ in cBN $\left.\left(\rho=3.488 \mathrm{~g} \mathrm{~cm}^{-3}\right)\right)$ results in $\approx 4 \%$ higher charge density $n_{\mathrm{c}}$ in diamond in comparison with $\mathrm{cBN}$.

As had been pointed out earlier [7] there are a number of limitations to a quantitative analysis of low-loss features. The most serious problems in this context arise from the uncertainty in apparent mass density of DLC films as a result of considerable fractions of hydrogen content, and phase overlap in the case of nanocrystalline heterostructures, particularly TiN, TiC and AlN in cBN based cutting tool materials all contributing to a collective bulk plasmon peak. Therefore the analysis of core-loss ELNES appears to be the more appropriate choice for a reliable quantification of $\mathrm{sp}^{2} / \mathrm{sp}^{3}$ ratios. Excitations of $1 \mathrm{~s}$ electrons in the ground state to vacant $\pi^{*}$ antibonding 
states results in characteristic sharp $\pi$ peaks observed in the regions $188<\Delta E<192 \mathrm{eV}$ and $282<\Delta E<286 \mathrm{eV}$ in the B-K and C-K ELNES, respectively. Excitations to the $\sigma^{*}$ states occur at higher energy levels. The ratio of integrated intensities under the $\pi$ and $\sigma$ peaks should approximately be proportional to the relative number of $\pi$ and $\sigma$ arbitals, i.e. $1: 3$ in $100 \% \mathrm{sp}^{2}$ and $0: 4$ in $100 \% \mathrm{sp}^{3}$ structures with four valence electrons per atom. It is assumed that the matrix element for $1 \mathrm{~s} \rightarrow \pi^{*}$ transitions is invariant for all carbon and BN allotropes, and overlap of $\pi$ and $\sigma$ peaks is negligible [7]. Direct quantification of the fraction of $\mathrm{sp}^{2}$ bonded atoms faces the problem of the choice of appropriate integration boundaries. Energy windows of 5 and $15 \mathrm{eV}$ as proposed for the integration of $\pi$ and $\sigma$ intensities for C-K [13] appear to be rather arbitrary and might result in considerable errors in both the carbon and $\mathrm{BN}$ system.

This difficulty is avoided by considering the normalized integrated intensity ratio of $\pi$ peaks in unknown forms of carbon or BN to those of $100 \% \mathrm{sp}^{2}$ bonded material, i.e. graphitized carbon and hBN, respectively. By using the integrated intensities $I(\Delta E)$ over sufficiently large energy windows $\Delta E$ as normalization factors [7], the fraction $\mathrm{f}$ of $\mathrm{sp}^{2}$ bonded atoms in an unknown carbon or $\mathrm{BN}$ material is given by the ratio

$$
f=\left(\frac{I_{\mathrm{u}}}{I_{\mathrm{o}}}\right)_{\pi}
$$

where $I_{\mathrm{u}}=\frac{I_{\mathrm{u} \pi}}{I_{\mathrm{u}}(\Delta E)}$ is the normalized intensity of the $\pi$ peak in the unknown material, and $I_{\mathrm{o}}=$ $\frac{I_{0 \pi}}{I_{0}(\Delta E)}$ is the normalized intensity of the $\pi$ peak in graphitized carbon or hBN reference material, respectively. For sufficiently large integration windows $\Delta E$ the fluctuations in intensity as an effect of ELNES features are levelled out and $f(\Delta E)$ is expected to converge to its true value, provided that plural scattering effects have been eliminated. However, equation (3) might be valid only in situations where both the unknown and reference material are in identical crystal orientations with respect to the momentum transfer vector, since in the highly anisotropic structures of graphite and hBN the relative intensity of the $\pi$-peak critically depends on the crystal orientation (Fig. 2). Plots of $f(\Delta E)$ versus integration windows $\Delta E$ are shown in Figure 7 for DLC and $\mathrm{BN}$ in the binder of $\mathrm{cBN}$ based tool material. In the case of amorphous DLC referenced to partially graphitized carbon the ratio $f(\Delta E)$ is apparently independent of specimen orientation and indicates $\approx 30 \% \mathrm{sp}^{2}$ bonding in DLC. The ratio $f(\Delta E)$ of the normalized $\pi$-peak from BN in the binder phase of $\mathrm{cBN}$ based material to the $\pi$-peak from $\mathrm{hBN}(q \perp c)$ indicates $\approx 50 \% \mathrm{sp}^{2}$ bonding. On the other hand referencing BN from the binder to hBN $(q \| c)$ would indicate a much lower fraction $\approx 15 \% \mathrm{sp}^{2}$ bonding in this material. Since the BN nanocrystallites are randomly oriented in the binder material, statistically the sampling from crystallites where $q$ is not parallel to the $c$-axis should make the largest contribution to the ELNES. Therefore it is argued that the intensity of the $\pi$-peak from the binder should be rather referenced to hBN $(q \perp c)$, thus a fraction $\lesssim 50 \% \mathrm{sp}^{2}$ bonding can be assumed for BN present in the binder phase. Due to the fact that the overall shape of the dominating $\sigma$-resonances in DLC and graphitized carbon, and BN in the binder and hBN reference material, respectively are similar to each other, the functions $f(\Delta E)$ converge rapidly to constant values for integration intervals $\Delta E>40 \mathrm{eV}$. Applying the law of mixtures, a ratio $\mathrm{sp}^{2} / \mathrm{sp}^{3} \approx 50 / 50$ for $\mathrm{BN}$ in the binder corresponds to an apparent density $\rho \approx 2.9 \mathrm{~g} \mathrm{~cm}^{-3}$ in good agreement with the data obtained from the experimental plasmon energies (Figs. 2a and 6).

On the other hand a ratio $\mathrm{sp}^{2} / \mathrm{sp}^{3} \approx 30 / 70$ corresponds to an apparent density $\rho \approx 3.1 \mathrm{~g} \mathrm{~cm}^{-3}$ for DLC. This value clearly falls outside the range expected for the carbon and BN systems (shaded area as indicated in Fig. 6) and would corresponds to a plasmon energy $E_{\mathrm{p}} \approx 31 \mathrm{eV}$, so far not observed in any DLC material. This discrepancy between $\mathrm{sp}^{2} / \mathrm{sp}^{3}$ ratio as derived from core- 


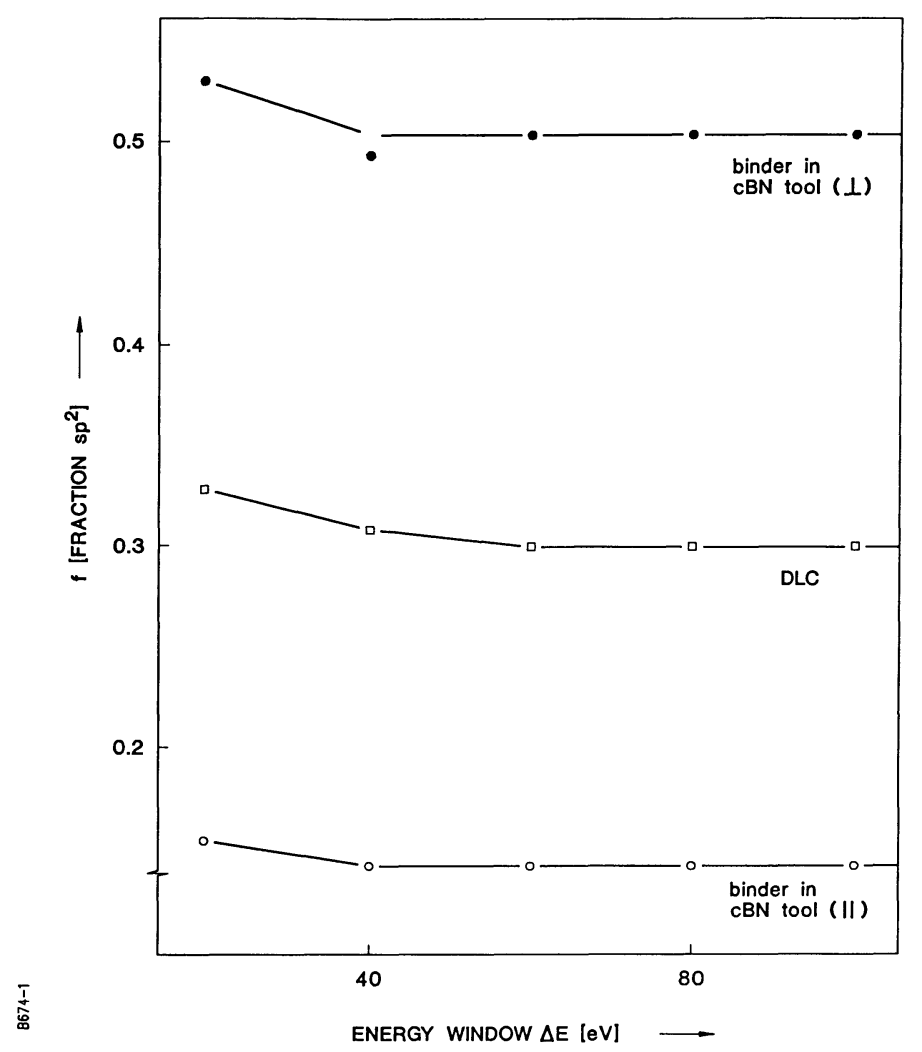

Fig. 7. - Plot of fraction $f(\Delta E)$ of $\mathrm{sp}^{2}$ bonded atoms in diamond-like carbon (DLC) and BN in binder of cBN based cutting tool material; momentum transfer vector parallel $(q \| c)$, and perpendicular $(q \perp c)$ to $c$-axis, respectively.

loss ELNES in amorphous DLC and their corresponding plasmon energy is attributed to a high fraction of hydrogen content (up to 50 at.\% H) in thin film DLC [19] resulting in low apparent density of this material.

\section{Summary and Conclusions}

As various technologies for the preparation of diamond, cBN, DLC and other superhard bulk materials and thin film coatings have advanced, a number of spectroscopy methods have emerged of which both Raman spectroscopy and EELS are indispensable tools for the characterization of these materials. Though Raman spectroscopy in principle is capable to identify various allotropic forms in carbon [20] and other systems, it often lacks the spatial resolution necessary particularly for phase identification in nanocrystalline heterostructures such as cBN based composites, so far accessible only by EELS techniques in TEM/STEM.

It has been confimed that the similarity in crystal symmetry between carbon and the isostructural BN system results in spectral similarities in both the low-loss features and core-loss ELNES. The analysis of these features provides characteristic fingerprints which allow unambiguous phase identification of carbon and $\mathrm{BN}$ allotropes. The major difference between $\mathrm{sp}^{3}$ and $\mathrm{sp}^{2}$ bonded al- 
lotropes in carbon and $\mathrm{BN}$ is manifested in a shift of plasmon peaks to lower energies in structures with the latter bonding type due to lower carrier charge density. The cubic polymorphs, $\mathrm{cD}$ and $\mathrm{cBN}$ give rise to characteristic humps at the low-energy slope of their respective plasmon peaks, whereas no such humps are observed in spectra of the hexagonal forms. Structural differences between the various allotropic forms are also manifested in their corresponding C-K and B-K ELNES characteristics. The relative intensities of peaks III and IV (Fig. 1b) in hD differ from those in $\mathrm{cD}$, whereas in wBN additional intensity at energy losses $\approx 209 \mathrm{eV}$ provide a clear fingerprint for identification of this phase.

The comparison of N-ELNES allows one to discriminate between $\mathrm{N}$ present in AlN, TiN, hBN and $\mathrm{cBN} / \mathrm{wBN}$. Similarly, C-K ELNES and B-K ELNES provide fingerprints for the identification of $\mathrm{C}$ in $\mathrm{B}_{4} \mathrm{C}$, $\mathrm{TiC}$ and amorphous carbon, and $\mathrm{B}$ in $\mathrm{TiB} / \mathrm{TiB}_{2}, \mathrm{~B}_{4} \mathrm{C}$ and amorphous borate glasses, respectively.

The observation of $\pi$ peaks in core-loss ELNES due to $1 \mathrm{~s} \rightarrow \pi^{*}$ transitions in graphitic carbon, DLC, hBN and cBN based cutting tool materials are characteristic of the presence of $\mathrm{sp}^{2}$ bonding in these materials. It is suggested that the fraction of $\mathrm{sp}^{2}$ bonding in mixed $\mathrm{sp}^{2} / \mathrm{sp}^{3}$ bonded DLC and $\mathrm{cBN}$ based tool material could be quantified from the ratio of the normalized intensity of the $\pi$ peaks in these materials to that of $100 \% \mathrm{sp}^{2}$ bonded reference material, i.e. graphitized carbon and hBN, respectively. Since the relative intensity of the $\pi$-peak in the highly anisotropic graphite and $\mathrm{hBN}$ depends on crystal orientation, quantification requires identical orientations of the caxis with respect to the direction of the momentum transfer vector and thus the incident electron beam in both the unknown and reference materials.

\section{References}

[1] Egerton R.F., Electron Energy-Loss Spectroscopy in the Electron Microscope (Plenum Press, New York, 1986).

[2] Howie A. and Walsh C., Microsc. Microanal. Microstruct. 2 (1991) 171-181.

[3] Brydson R., Sauer H., Engel W. and Zeitler E., Microsc. Microanal. Microstruct. 2 (1991) 159-169.

[4] Hofer F. and Golob P., Ultramicroscopy 21 (1987) 379-384.

[5] Joy D.G. and Maher D.M., Ultramicroscopy 3 (1978) 69-74.

[6] Schmid H.K., Proc. 13th ICEM, Paris (1994) 695-96.

[7] Berger S.D., McKenzie D.R. and Martin P.J., Phil. Mag. Lett. 57 (1988) 285-290.

[8] Egerton R.F. and Whelan M.J., Phil. Mag. 30 (1974) 739-749.

[9] Kurdyumov A.V., Ostrovskaya N.F. and Golubev A.S., Sverkhtverdye Mater. 6 [4] (1984) 17-25.

[10] Saito Y., Suzuki N., Shinohara H., Hayashi T. and Tomita M., Ultramicroscopy 41 (1992) 1-9.

[11] Rosenberg R.A., Love P.J. and Rehn V., Phys. Rev. B 33 (1986) 4034.

[12] Kulik J., Lifshitz Y., Lempert G.D., Rotter S., Rabalais J.W. and Marton D., Proc. 50th Meeting Electr. Microsc. Soc. Am. (1992) 1272-73.

[13] Cuomo J.J., Doyle J.P., Bryley J. and Liu J.C., Appl. Phys. Lett. 58 (1991) 466-468.

[14] Hosoi J., Oikawa T. and Inoue M., J. Electr. Spectr. Relat. Phenom. 27 (1982) 243-254.

[15] Disko M.M., Krivanek O. and Spence J.C.H., Proc. 39th EMSA Conf. (1981) 192-93.

[16] Schmid H.K., Proc. Electr. Microsc. Soc. South. Afr. 23 (1993) 7-9, ibid. 78.

[17] McColm I.J., Ceramic Hardness (Plenum Press, NY, 1990) p. 209.

[18] Rapoport E., Ann. Chim. Fr. 10 (1985) 607-638.

[19] Diamond and Diamond-like Carbon Coatings: Gorham Adv. Mat. Inst., Vol. 1 (1989).

[20] Knight D.S. and White W.B., J. Mater. Res. 4 (1989) 385-393. 